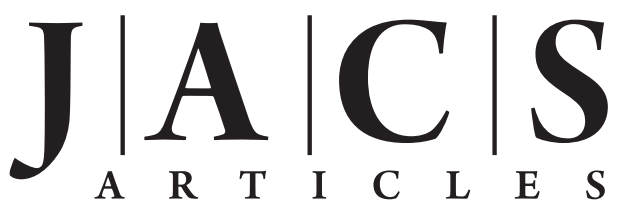

Published on Web 06/29/2010

\title{
Crystalline Domain Structure and Cholesterol Crystal Nucleation in Single Hydrated DPPC:Cholesterol:POPC Bilayers
}

\author{
Roy Ziblat, ${ }^{\dagger}$ Leslie Leiserowitz, ${ }^{* \neq}$ and Lia Addadi ${ }^{*, \dagger}$ \\ Department of Structural Biology and Department of Materials and Interfaces, Weizmann \\ Institute of Science, 76100 Rehovot, Israel
}

Received May 10, 2010; E-mail: leslie.leiserowitz@weizmann.ac.il; lia.addadi@weizmann.ac.il

\begin{abstract}
Grazing incidence X-ray diffraction measurements were performed on single hydrated bilayers and monolayers of DPPC:Cholesterol:POPC at varying concentrations. There are substantial differences in the phase and structure behavior of the crystalline domains formed within the bilayers relative to the corresponding monolayers, due to interactions between the opposing leaflets. Depending on the lipid composition, these interactions led to phase separation, changes in molecular tilt angle, or formation of cholesterol crystals. In monolayers, DPPC and cholesterol form a single crystalline phase at all compositions studied. In bilayers, a second crystalline phase appears when cholesterol levels are increased: domains of cholesterol and DPPC form monolayer thick crystals where each of the lipid leaflets diffracts independently, whereas excess cholesterol forms cholesterol bilayer thick crystals at a DPPC:Chol ratio $<46: 54 \pm 2$ mol $\%$. The nucleation of the cholesterol crystals occurs at concentrations relevant to the actual cell plasma membrane composition.
\end{abstract}

\section{Introduction}

Cell membranes possess lipid domains with different compositions and structures, which are important to cell functionality. ${ }^{1-4}$ Lipid domains were studied extensively during the past two decades. Several characteristics of the lipid molecules govern their phase and packing structure behavior in the lipid domains, including their chemical structure, molecular charge, and stereochemistry. There is specifically a growing interest in the interactions between the opposing leaflets forming the lipid bilayer. ${ }^{5-10}$ This study provides insights into such interactions in symmetrical artificial bilayers composed of cholesterol and 1,2-dipalmitoyl-sn-glycero-3-phosphocholine (DPPC), specifically on the crystalline domains that exist in the lipid bilayer.

Cell membranes contain a large variety of lipid domains, some of which are referred to as "lipid rafts", because of their relative rigidity and stability. These were first studied through the detergent resistant membrane (DRM) phenomenon. ${ }^{11-13} \mathrm{Com}-$

\footnotetext{
Department of Structural Biology.

Department of Materials and Interfaces.

(1) Simons, K.; Van Meer, G. Biochemistry 1988, 27 (17), 6197-6202.

(2) Simons, K.; Ikonen, E. Nature 1997, 387 (6633), 569-572.

(3) Simons, K.; Toomre, D. Nat. Rev. Mol. Cell Biol. 2000, 1 (1), 31-39.

(4) Hanzal-Bayer, M. F.; Hancock, J. F. FEBS Lett. 2007, 581 (11), 2098 104.

(5) Collins, M. D. Biophys. J. 2008, 94 (5), L32-L34.

(6) Goni, F. M.; Alonso, A.; Bagatolli, L. A.; Brown, R. E.; Marsh, D.; Prieto, M.; Thewalt, J. L. Biochim. Biophys. Acta 2008, 1781 (1112), 665-684.

(7) Wan, C.; Kiessling, V.; Tamm, L. K. Biochemistry 2008, 47 (7), 21902198.

(8) Bennett, W. F. D.; MacCallum, J. L.; Hinner, M. J.; Marrink, S. J.; Tieleman, D. P. J. Am. Chem. Soc. 2009, 131 (35), 12714-12720.

(9) Collins, M. D.; Keller, S. L. Biophys. J. 2007, 15a-15a.

(10) Collins, M. D.; Keller, S. L. Proc. Natl. Acad. Sci. U.S.A. 2008, 105 (1), 124-128.

(11) Brown, D. A.; London, E. Biochem. Biophys. Res. Commun. 1997, $240(1), 1-7$.
}

parisons between the lipid composition of the original membrane and its DRMs showed elevation in cholesterol (Chol) and sphingomyelin (SM) concentrations in the latter. This observation was explained by considering that the sphingolipids in the cell membrane have a larger portion of saturated alkyl chains relative to the glycerolipids; however, the DRMs also contain a significant amount of saturated glycerolipids. ${ }^{12}$ Glycerophosphocholines are the most abundant lipid molecules in mammalian cells. ${ }^{14}$ It is thus important to study domain mixtures of cholesterol and glycerolipids, as well as cholesterol and sphingolipids. Several studies have shown that DPPC, a saturated glycerolipid with a phosphocholine headgroup, forms mixed phases and ordered domains with cholesterol. ${ }^{15-21}$

The phases formed by lipid assemblies are generally sorted into three main groups, ${ }^{6,22}$ depending on the melting temperature; Liquid disordered $\left(l_{d}\right)$ phases are characterized by high diffusion rates. Solid ordered $\left(\mathrm{S}_{\mathrm{o}}\right)$ or gel phases have a very low diffusion rate and are generally composed of lipids with

(12) Fiedler, K.; Kobayashi, T.; Kurzchalia, T. V.; Simons, K. Biochemistry 1993, 32 (25), 6365-6373.

(13) Arnulphi, C.; Sot, J.; Garcia-Pacios, M.; Arrondo, J. L. R.; Alonso, A.; Goni, F. M. Biophys. J. 2007, 93 (10), 3504-3514.

(14) van Meer, G.; Voelker, D. R.; Feigenson, G. W. Nat. Rev. Mol. Cell Biol. 2008, 9 (2), 112-124.

(15) Estep, T. N.; Mountcastle, D. B.; Barenholz, Y.; Biltonen, R. L.; Thompson, T. E. Biochemistry 1979, 18 (10), 2112-2117.

(16) Ratajczak, M. K.; Ege, C.; Ko, Y. T. C.; Majewski, J.; Kjaer, K.; Lee, K. Y. C. Biophys. J. 2005, 88 (1), 73a-73a.

(17) Karmakar, S.; Raghunathan, V. A.; Mayor, S. J. Phys.: Condens. Matter 2005, 17 (14), S1177-S1182.

(18) Stottrup, B. L.; Keller, S. L. Biophys. J. 2006, 90 (9), 3176-3183.

(19) Marsh, D. Biochim. Biophys. Acta 2009, 1788 (10), 2114-2123.

(20) Bach, D.; Wachtel, E. Biochim. Biophys. Acta 2003, 1610 (2), 187197.

(21) Ivankin, A.; Kuzmenko, I.; Gidalevitz, D. Phys. Rev. Lett. 2010, 104 (10), 108101

(22) Feigenson, G. W. Biochim. Biophys. Acta 2009, 1788 (1), 47-52. 
saturated alkyl chains. Liquid ordered $\left(l_{0}\right)$ phases are characterized by an intermediate diffusion coefficient and may contain high levels of cholesterol. The nature of the domains formed in the phases described above are defined by their lipid composition, and their sizes can grow to micrometer scale under equilibrium conditions. ${ }^{23}$ The $\mathrm{l}_{\mathrm{o}}$ and $\mathrm{S}_{\mathrm{o}}$ phases include crystalline domains detectable by X-ray diffraction. ${ }^{16,24-29}$ Their coherence lengths are typically nanometers in the $1_{0}$ phase and tens of nanometers in the $S_{o}$ phase.

Grazing incidence X-ray diffraction (GIXD) is a method developed to probe the structure of two-dimensional crystals and can yield direct information on the organization of laterally ordered lipid sheets. ${ }^{30,31}$ To enable the measurement of the thin flat samples, the incidence X-ray beam is set at a grazing angle, to enhance the diffraction signal from the sample relative to scattering from its supporting substrate. These studies are generally performed on artificial lipid monolayers at the air/ water interface. Monolayers differ, however, from the single lipid bilayer constituents of the cell membrane, especially because the hydrophobic parts of the molecules are exposed to air and therefore do not interact with an opposing lipid leaflet. In bilayers, it is thus of fundamental interest to study the interactions between opposing leaflets, which were experimentally shown to influence membrane domain formation in model membranes. ${ }^{5,7}$ Interdigitation of alkyl chains in the bilayer core, i.e. at the interface between the two lipid leaflets, was also suggested to occur. ${ }^{32}$

GIXD measurements on single hydrated lipid bilayers were only recently conducted to successfully reveal crystalline order. $^{33-35}$ In our work ${ }^{34}$ GIXD was performed on a single hydrated bilayer composed of SM:Chol:POPC, while maintaining the sample close to the dew point, such that the bilayer is completely hydrated. We study here, using the same method, the structure of lipid bilayers of DPPC:Chol:POPC, and we analyze the interactions between the opposing leaflets.

The cholesterol molecule participates in membrane structure and determines its mechanical and dynamical properties. Cholesterol is believed to be an essential component in the formation of functional ordered domains. ${ }^{2}$ Its concentration was found to be as high as $20-45 \%$ of the phospholipids in the plasma membrane in mammalian cells. ${ }^{14,36}$ When accumulated

(23) Veatch, S. L.; Keller, S. L. Biophys. J. 2003, 85 (5), 3074-3083.

(24) Vaknin, D.; Kelley, M. S. Biophys. J. 2000, 79 (5), 2616-2623.

(25) Majewski, J.; Kuhl, T. L.; Kjaer, K.; Smith, G. S. Biophys. J. 2001, 81 (5), 2707-2715.

(26) Tristram-Nagle, S.; Liu, Y. F.; Legleiter, J.; Nagle, J. F. Biophys. J. 2002, 83 (6), 3324-3335.

(27) Lee, K. Y. C. In CNLS conference, Santa Fe, New Mexico, May 21, 2007; Santa Fe, New Mexico, 2007.

(28) Mills, T. T.; Huang, J. Y.; Feigenson, G. W.; Nagle, J. F. Gen. Physiol. Biophys. 2009, 28 (2), 126-139.

(29) Ratajczak, M. K.; Chi, E. Y.; Frey, S. L.; Cao, K. D.; Luther, L. M.; Lee, K. Y. C.; Majewski, J.; Kjaer, K. Phys. Rev. Lett. 2009, 103 (2), 028103.

(30) Als-Nielsen, J.; Jacquemain, D.; Kjaer, K.; Leveiller, F.; Lahav, M.; Leiserowitz, L. Phys. Rep. 1994, 246 (5), 252-313.

(31) Jensen, T. R.; Kjaer, K. In Novel methods to study interfacial layers; Moebius, D., Miller, R., Eds.; Elsevier: Amsterdam, 2001; pp 205254.

(32) Pinto, S. N.; Silva, L. C.; de Almeida, R. F. M.; Prieto, M. Biophys. J. 2008, 95 (6), 2867-2879.

(33) Miller, C. E.; Majewski, J.; Watkins, E. B.; Mulder, D. J.; Gog, T.; Kuhl, T. L. Phys. Rev. Lett. 2008, 1 (5), 058103.

(34) Ziblat, R.; Kjaer, K.; Leiserowitz, L.; Addadi, L. Angew. Chem., Int. Ed. 2009, 48 (47), 8958-8961.

(35) Watkins, E. B.; Miller, C. E.; Mulder, D. J.; Kuhl, T. L.; Majewski, J. Phys. Rev. Lett. 2009, 102 (23), 238101.

(36) Ikonen, E. Nat. Rev. Mol. Cell Biol. 2008, 9 (2), 125-138. by cells in excess, cholesterol was found to participate in several pathological phenomena ${ }^{37,38}$ such as cataract ${ }^{39}$ and atherosclerosis. ${ }^{40}$ Large amounts of cholesterol monohydrate crystals are found in gall stones ${ }^{41}$ and foam cells ${ }^{42}$ at the site of atherosclerotic plaques. It seems that these crystals are an early cause rather than a late outcome of atherosclerosis inflammation. ${ }^{43}$ The nucleation mechanism by which cholesterol crystals are formed in and around cells is not well understood. It was recently shown that cholesterol enriched macrophage cells form cholesterol crystals near the cytoplasm membrane, ${ }^{44}$ and it was suggested that these crystals nucleate at the lipid membrane. ${ }^{20,40,45}$ We hereby demonstrate that the nucleation of cholesterol crystals can occur within DPPC:Chol:POPC bilayers and discuss the conditions for nucleation.

\section{Experimental Section}

Bilayer Deposition. Silicon wafers (SESO, France) were treated with $n$-hexane, acetone, ethanol; left in Piranha for $20 \mathrm{~min}$; and washed. Deposition of a first polymer cushion is by immersion of wafers in $1000 \mathrm{ppm}$ polyethyleneimine (PEI) overnight. The minimum thickness of this layer is estimated to be $5 \mathrm{~nm} .{ }^{46}$ The desired proportion of 1,2-dipalmitoyl-sn-glycero-3-phosphocholine (DPPC), 1-palmotoyl-2-oleoyl-sn-glycero-3-phosphocholine (POPC) (Avanti Polar Lipids), and cholesterol (>99\% Sigma-Aldrich) was dissolved in chloroform and spread $(\sim 50 \mu \mathrm{L})$ on a preheated Langmuir trough. Prior to spreading, cholesterol was purified by crystallization. ${ }^{47}$ The temperature of the Langmuir trough was set to be higher than the melting temperature of the lipid composition, when possible, typically above $40-50^{\circ} \mathrm{C}$. After $10 \mathrm{~min}$ (chloroform evaporation time) the lipid monolayer at the air-water interface is compressed to $30 \mathrm{mN} / \mathrm{m}$. (Figure 1) The water is then drained out of the trough, lowering the lipid monolayer on top of a silicon/ quartz wafer covered with a polymer cushion which lies at the bottom of the trough. Some water remains sandwiched between the wafer and the lipid monolayer (Figure 1). This layer, though covered with a lipid monolayer, undergoes a slow evaporation. Once the water has almost completely evaporated, the wafer is lifted and placed upside down on top of a water-filled Teflon plate, placed in advance at the bottom of the trough, and now covered with a lipid monolayer as well. Once placed on the Teflon plate, the wafer is gradually cooled down to $4{ }^{\circ} \mathrm{C}$ and kept so until AFM or GIXD experiments are performed.

Prior to the GIXD experiment a second PEI cushion layer is deposited on top of the bilayer by immersion in $1000 \mathrm{ppm}$ PEI for $10 \mathrm{~min}$ and washed. Water comprises $85 \%$ of the PEI cushion volume. ${ }^{46}$ The bilayer sample is constantly kept under either water or high humidity. After deposition of the PEI layer, the contact angle of the bilayer is $0^{\circ}$, while in the absence of PEI the contact angle is hydrophilic but higher than $30^{\circ}$. Samples which contain

(37) Ikonen, E. Physiol. Rev. 2006, 86 (4), 1237-1261.

(38) Maxfield, F. R.; Tabas, I. Nature 2005, 438 (7068), 612-621.

(39) Cenedella, R. J. JAMA, J. Am. Med. Assoc. 1987, 257 (12), 16021602.

(40) Mason, R. P.; Tulenko, T. N.; Jacob, R. F. Biochim. Biophys. Acta 2003, 1610 (2), 198-207.

(41) Weihs, D.; Schmidt, J.; Goldiner, I.; Danino, D.; Rubin, M.; Talmon, Y.; Konikoff, F. M. J. Lipid Res. 2005, 46 (5), 942-948.

(42) Tulenko, T. N.; Chen, M.; Mason, P. E.; Mason, R. P. J. Lipid Res. 1998, 39 (5), 947-956.

(43) Duewell, P.; et al. Nature 2010, 464 (7293), 1357-1361.

(44) Ong, D. S.; Anzinger, J. J.; Leyva, F. J.; Rubin, N.; Addadi, L.; Kruth, H. S. J. Lipid Res. 2010, in press.

(45) Rapaport, H.; Kuzmenko, I.; Lafont, S.; Kjaer, K.; Howes, P. B.; AlsNielsen, J.; Lahav, M.; Leiserowitz, L. Biophys. J. 2001, 81 (5), 27292736.

(46) Wong, J. Y.; Majewski, J.; Seitz, M.; Park, C. K.; Israelachvili, J. N.; Smith, G. S. Biophys. J. 1999, 77 (3), 1445-1457.

(47) Perl-Treves, D.; Kessler, N.; Izhaky, D.; Addadi, L. Chem. Biol. 1996, 3 (7), 567-77. 

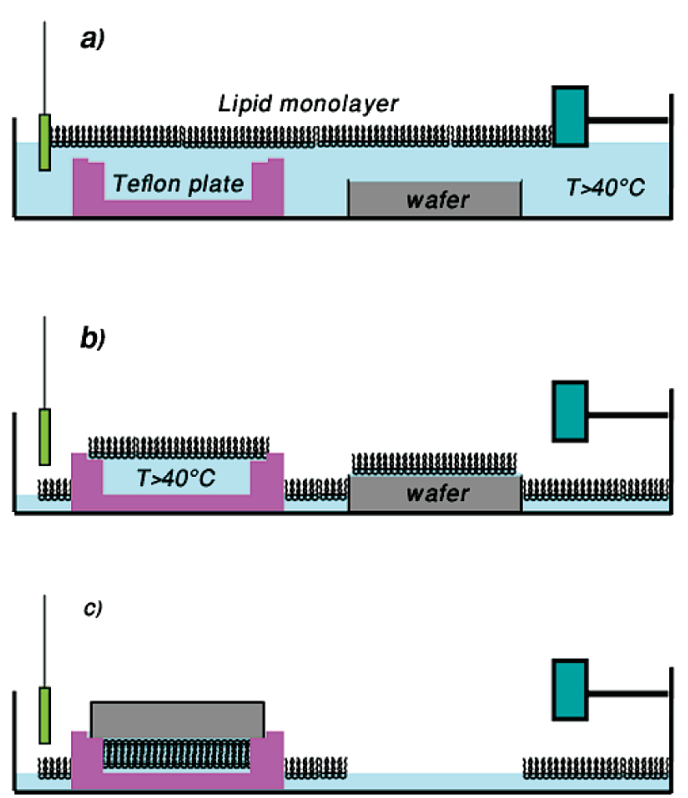

Figure 1. Schematic representation of the bilayer deposition process. (a) Lipid molecules are spread at the air-water interface and compressed to a surface pressure of $30 \mathrm{mN} / \mathrm{m}$. (b) Water is drained, and a lipid monolayer is deposited on top of both a preplaced wafer and Teflon plate. (c) The wafer is lifted and placed upside down on top of the Teflon plate to form a lipid bilayer. An $\sim 1 \mathrm{~mm}$ thick water layer remains between the bilayer and the Teflon plate. The entire deposition process is performed at a temperature $>45^{\circ} \mathrm{C}$.

cholesterol molecules remain intact for long periods when $10 \%$ POPC (1-palmitoyl-2-oleoyl-sn-glycero-3-phosphocholine) is added to the mixture. POPC separates from cholesterol and $\mathrm{DPPC}^{6}$ and does not participate in the crystalline domain structures, because of the presence of one kinked acyl chain.

AFM. AFM scans were performed with a BIOSCOPE (Veeco) or a Molecular Imaging PICO (Agilent Technologies) system under wet contact or tapping mode, using Silicon Nitride tips (Digital Instruments). Scan frequencies were typically $2-3 \mathrm{~Hz}$, and a line resolution of either 256 or 512 was used.

Humidity GIXD Setup. A humidity chamber was designed and constructed at the Weizmann Institute and adapted to be mounted on beamline BW1 at the DORIS III Synchrotron DESY (Hamburg, Germany). The methodology was recently introduced and described. ${ }^{34}$ Briefly, the sample is completely wet when placed inside the humidity chamber. A controlled evaporation process is then initiated to enable GIXD measurements. The temperature of the chamber walls is controlled by a circulating flow from a refrigerated bath, and the sample temperature is adjusted by a Peltier element. The chamber is filled with humidified helium. Relative humidity, gas temperature, and sample temperature are monitored and maintained by PID feedback loops. The measurements are performed under continuous control of temperature and humidity. Although this setup was designed for bilayer studies, it can be used on any two-dimensional crystal which requires hydration.

GIXD Measurements. A monochromatic X-ray beam $(\lambda=$ $1.304 \AA$ ) was adjusted to strike the sample surface at an incident angle $\alpha_{i}=0.85 \alpha_{c}$, where $\alpha_{c}$ is the critical angle for total external reflection for the $\mathrm{He}$-silicon or the $\mathrm{He}$ - water interface for bilayer and monolayer setups respectively. The dimensions of the footprint of the incoming X-ray beam on the sample surface were $1 \mathrm{~mm} \times$ $36 \mathrm{~mm}$ for silicon and $1 \mathrm{~mm} \times 50 \mathrm{~mm}$ for water. GIXD signals were obtained from 2-D crystallites randomly oriented about the wafer surface normal in the form of a 2-D "powder". The scattered intensity was collected by means of a vertical position-sensitive detector, measuring $q_{z} \approx 2 \pi \sin \left(\alpha_{\mathrm{f}}\right) / \lambda$, which is the out-of-plane component of the scattering vector. Measurements were performed by scanning the horizontal component, $q_{x y} \approx 4 \pi \sin \left(2 \theta_{x y}\right) / \lambda$, of the scattering vector, where $2 \theta_{x y}$ is the angle between the incident and diffracted beam projected onto the horizontal plane. The diffraction data are represented in two ways: (1) Bragg peaks $I\left(q_{x y}\right)$ are the integral of the GIXD pattern $I\left(q_{x y}, q_{z}\right)$ over $q_{z}$; (2) Bragg rod intensity profiles $I\left(q_{z}\right)$ are the scattered intensity $I\left(q_{x y}, q_{z}\right)$ integrated across the $q_{x y}$ range of each Bragg peak. The $q_{x y}$ positions of the Bragg peaks yield the lattice repeat distances $d=2 \pi / q_{x y}$, which may be indexed by the two Miller indices $h, k$ to yield the unit cell. The full width at half-maximum (fwhm) of the Bragg peaks yields the lateral 2-D crystalline coherence length $L_{x y} \approx 0.9(2 \pi) / \mathrm{fwhm}\left(q_{x y}\right)$. The Bragg rod profile along $q_{z}$ similarly gives a measure of the thickness of the crystalline film. Control studies on monolayers deposited at the air/water interface were performed at the same beamline using a liquid surface diffractometer. ${ }^{31,48}$ All measurements were performed at sample temperatures of $\sim 6-7{ }^{\circ} \mathrm{C}$.

\section{Results}

Bilayer samples were deposited at the HASYLAB synchrotron facility using a Langmuir trough (Figure 1). From the moment of deposition and until the end of the measurements the sample is kept constantly under water. When placed inside the humidity chamber for GIXD measurements, the sample is covered with an $\sim 1 \mathrm{~mm}$ thick water layer, which subsequently undergoes evaporation under controlled conditions. Scattering calculations show that when the thickness of the condensed water layer on top of the lipid bilayer is lower than $1 \mu \mathrm{m}$, GIXD measurements can be successfully performed.

Throughout the diffraction measurements the sample is kept under high humidity ( $>94 \%)$. To avoid even local and temporary drying of the sample, the lipid bilayer is covered with a polyethyleneimine (PEI) layer with a thickness of a few to tens of nanometers. The highly hydrophilic PEI layer, deposited prior to GIXD measurements, adsorbs water guaranteeing the presence of a water reservoir that does not evaporate even at humidity $<100 \%$. Prior to extraction from the humidity chamber, a thick water layer is condensed on top of the sample. Samples are then characterized to ensure that no damage occurred to the bilayer sheet, during the process.

The conventional method to confirm the thickness of thin layer samples that are measured by GIXD is X-ray reflectivity. In the case of the hydrated lipid bilayer the sample is composed of a large number of blocks, including silicon, silicon oxide, polymer cushion, and the lipid bilayer that is composed of at least four varying electron density blocks, followed by a second polymer cushion. In addition to this complicated electron density profile, the top layer of water on the sample may vary over time according to relative humidity oscillations, all together making reflectivity scans an impractical method. In our case, therefore, AFM scans are deemed more reliable. ${ }^{34}$ We note that the lipid bilayer samples were deposited at relatively high temperature $\left(45-50{ }^{\circ} \mathrm{C}\right.$ ) but were imaged by AFM (Figure 2) at ambient temperature. The surface pressure/area isotherms of DPPC at these two temperatures are significantly different, the area per molecule at any given pressure being higher at $45^{\circ} \mathrm{C}$ than at ambient $T$. Therefore, bilayer samples, which were deposited at high temperature and scanned at ambient $T$, contain empty regions at the boundaries of which the height profile of the deposited layer can be easily evaluated. The PEI cushion, which consists mostly of water, is soft and is easily displaced by the pressure of the tip; therefore, its effect on the height image measured by AFM is negligible. The AFM tip is,

(48) Als-Nielsen, J.; Jacquemain, D.; Kjaer, K.; Leveiller, F.; Lahav, M.; Leiserowitz, L. Phys. Rep. 1994, 246 (5), 252-313. 

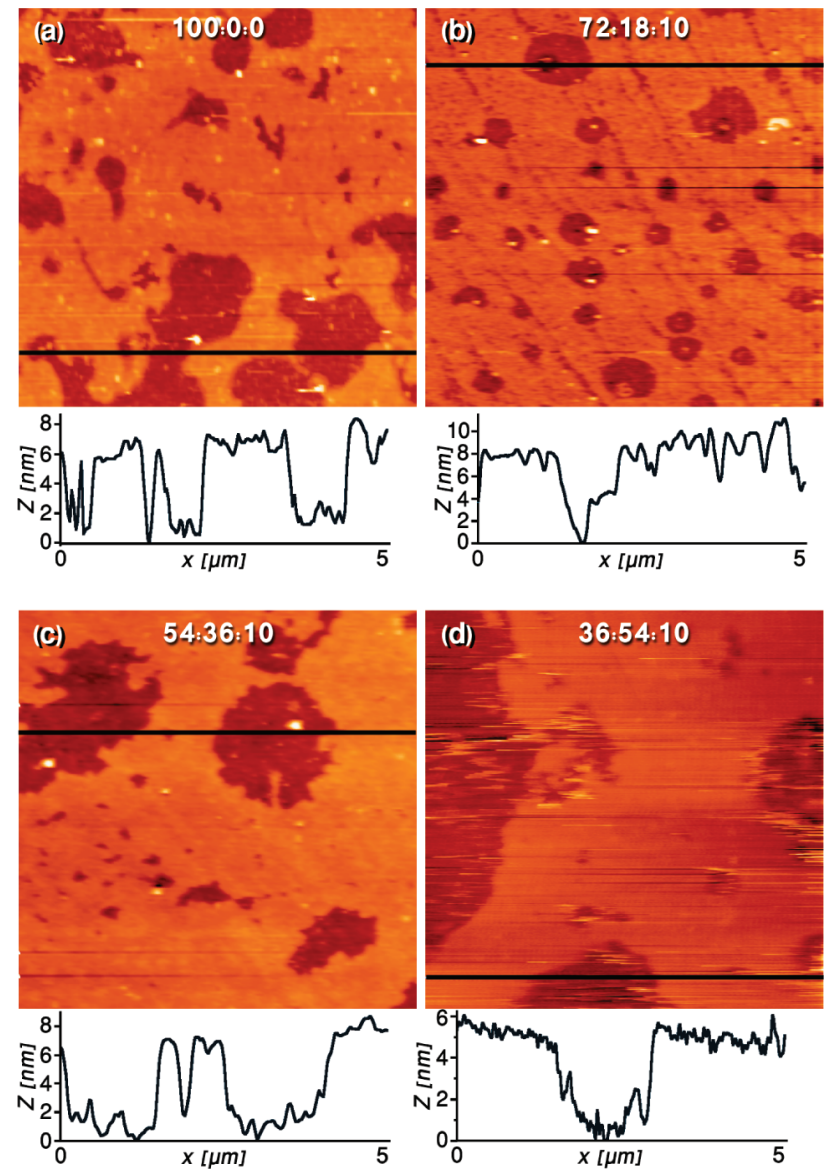

Figure 2. AFM bioscope height images, measured in wet contact mode Each scan is $5 \mu \mathrm{m} \times 5 \mu \mathrm{m}$, and the $\mathrm{z}$ color range is between 0 and $25 \mathrm{~nm}$. Images were linearly flattened. The AFM scans are performed after GIXD experiments. Each sample is composed of DPPC:Chol:POPC composition according to (a) 100:0:0, (b) 72:18:10, (c) 54:36:10, and (d) 36:54:10. Black lines on the AFM images represent the locations of height profile cuts which are given at the bottom of each scan image. These profiles, 5-6 nm thick, correspond to a bilayer.

however, forced through the polymer, thus introducing some noise to the scan images. The height profiles of the images obtained from DPPC samples after GIXD measurements consistently show a sample thickness of 5-6 nm, corresponding to a single bilayer (Figure 2). Each sample was scanned at 3-5 arbitrary locations, millimeters apart. Unlike bilayers, the height profiles of supported wet monolayers display a thickness of $\sim 1-1.5 \mathrm{~nm}$ and a pronounced roughness (Figure S1). AFM studies show that, in lipid bilayers which were not always fully hydrated, the bilayer structure is irreversibly damaged, and this is reflected in the AFM scans.

GIXD measurements were performed on both bilayer and monolayer systems with the following DPPC:Chol:POPC compositions: 100:0:0, 72:18:10, 54:36:10, and 36:54:10. POPC is introduced only to extend the bilayer stability in time but does not mix with cholesterol and DPPC. Figure 3 shows the Bragg peaks of bilayers and monolayers, extracted from the diffraction data. The intensities of the Bragg peaks were independently normalized for convenience (the un-normalized data is shown in Figure S2). Each of the GIXD scans is composed of several diffraction peaks. Data were fitted using Voight functions, and the separated peaks are shown in Figure $3 \mathrm{c}, \mathrm{d}$ for bilayer and monolayer accordingly.
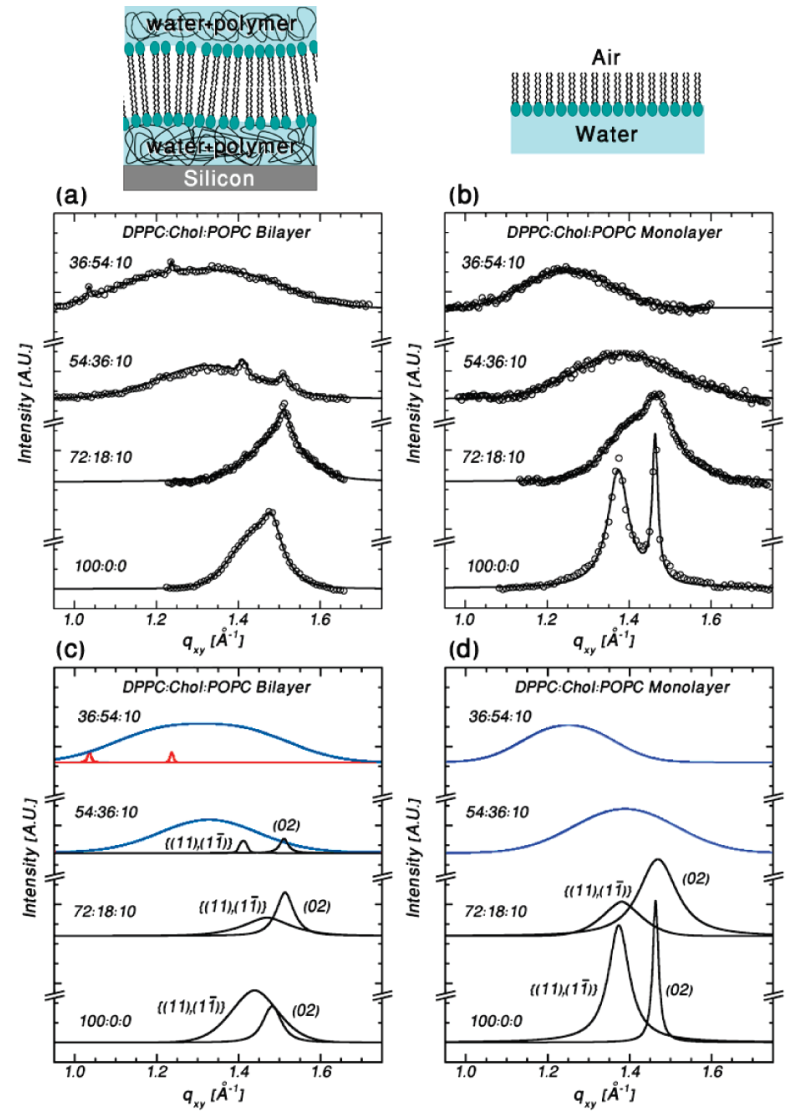

Figure 3. Bragg peak data from (a) bilayers and (b) monolayers at several DPPC:Chol:POPC compositions. Representative schemes of the samples are shown on top. The Bragg peaks were fitted with Voight functions and are shown after deconvolution: (c) bilayers; (d) monolayers. Peaks were colored according to their phases: DPPC phase (black), mixed DPPC:Chol phase (blue), and Chol monohydrate bilayer phase (red). The intensities of all Bragg peaks were factorized for easier comparison. Most DPPC Bragg peaks obey the multiplicity rule where $\{(11),(11)\}$ and $(02)$ have a ratio of integrated intensities of $\sim 1$. The 100:0:0 bilayer has a $10^{\circ}$ undulation which widens the $\{(11),(1 \overline{1})\}$ peak altering the ratio.

The lipid samples form several phases, all of which were identified previously by GIXD on a Langmuir trough, ${ }^{16,21,49}$ i.e. at the air/water interface. For convenience, the separated Bragg peaks are colored according to their phases: pure DPPC (black), mixed DPPC:Chol (blue), and pure Chol (red). For the 100:0:0 and 72:18:10 compositions both the monolayer and the bilayer samples show one crystalline phase. At 72:18:10 the GIXD patterns of the bilayer and the monolayer are similar, and thus also are their molecular arrangements, yet the molecular tilt, deduced from the fwhm of their Bragg rods (Figure 4a,b), is distinctively lower in the bilayer system. The molecular tilt in the monolayer is $26^{\circ}$ whereas in the bilayer it is $5^{\circ}$ (Table 1). Increasing the concentration of cholesterol to $36 \%$ yields in the monolayer a new wide peak which belongs to the DPPC:Chol mixed phase, positioned at $q_{x y}=1.39 \AA^{-1}$. In the bilayer, however, even though the composition is identical to that of the monolayer, two crystalline phases coexist. The position of the mixed phase peak at $q_{x y}=1.33 \AA^{-1}$ is distinctly lower than that in the monolayer sample. This shift to lower $q_{x y}$ is indicative of a lower DPPC:Chol ratio in the mixed phase, ${ }^{21,29,50}$ which

(49) Solomonov, I.; Weygand, M. J.; Kjaer, K.; Rapaport, H.; Leiserowitz, L. Biophys. J. 2005, 88 (3), 1809-1817.

(50) Ege, C.; Ratajczak, M. K.; Majewski, J.; Kjaer, K.; Lee, K. Y. C. Biophys. J. 2006, 91 (1), L1-L3. 

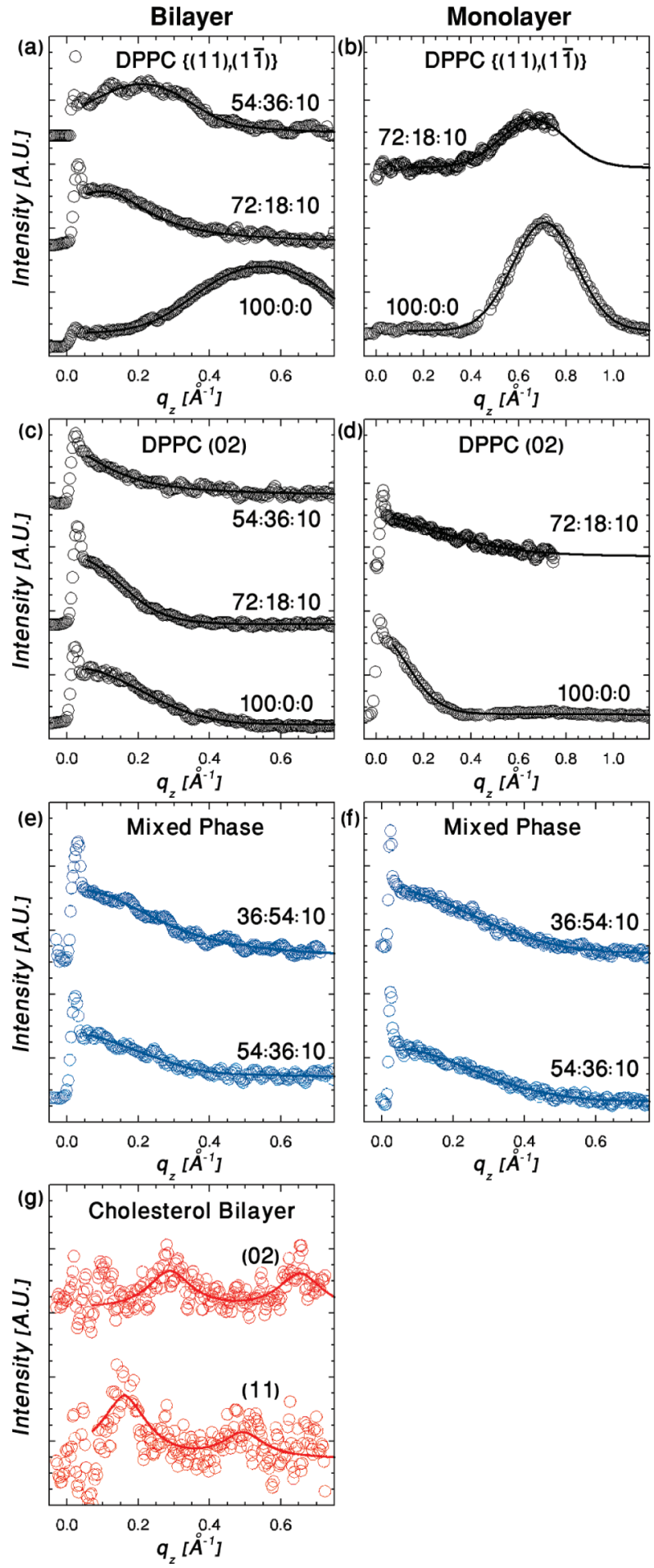

Figure 4. Bragg rods data from (a, c, e, g) bilayers and (b, d, f) monolayers at several DPPC:Chol:POPC compositions; $(\mathrm{a}-\mathrm{d})$ DPPC phase; (e-f) mixed DPPC:Chol phase; (g) cholesterol bilayer phase. The Bragg rods are colored according to their Bragg peaks in Figure 3. All Bragg rods show monolayer thick crystals. The Bragg rod of the 100:0:0 bilayer sample is wider relative to the 100:0:0 monolayer, due to an $\sim 10^{\circ}$ undulation of the bilayer sheet. The cholesterol Bragg rod shows a 34 Å thick crystal corresponding to a bilayer.

is in agreement with the observed formation of a second crystalline phase composed mainly of DPPC, identified by two Bragg peaks located at $q_{x y}=1.41,1.51 \AA^{-1}$.

At the increased cholesterol concentration of $54 \%$ the GIXD pattern also shows a different phase behavior between the monolayer and the bilayer. The monolayer yields one broad peak, which belongs to a mixed phase. Its position at $q_{x y}=$ $1.25 \AA^{-1}$ indicates that the monolayer has a higher cholesterol concentration than the monolayer composed of $36 \%$ cholesterol. On the other hand, the position of the broad mixed phase peak in the bilayer is at $q_{x y}=1.31 \AA^{-1}$, indicating that the domains formed by the mixed phase in the bilayer have a higher DPPC: Chol ratio than those observed in the monolayer. The remaining cholesterol gives rise to the two sharp Bragg peaks located at $q_{x y}=1.04,1.23 \AA^{-1}$, which belong to a crystalline cholesterol phase with a high coherence length of $60 \mathrm{~nm}$.

As a bilayer, the exocyclic chains of the cholesterol molecules are partially interdigitated, leading to a well packed structure with a high coherence length and a rectangular $10 \times 7.5 \AA^{2}$ unit cell. ${ }^{49}$ This unit cell structure corresponds to the bilayer of the macroscopically metastable phase of Chol $\cdot \mathrm{H}_{2} \mathrm{O} .{ }^{49}$ The Bragg rods of the GIXD peaks (Figure $4 \mathrm{~g}$ ), which are fingerprint evidence of the bilayer motif, yield a thickness of $35 \pm 1.3 \AA$, deduced from their fwhm. In contrast, the Bragg rods from the mixed DPPC:Chol and pure DPPC structural films, shown in Figure $4 \mathrm{a}-\mathrm{f}$, belong to crystalline phases with thicknesses of less than $20 \AA$ corresponding to monolayers. The position along $q_{z}$ of the Bragg rods of all the mixed phases show that the molecules are aligned essentially perpendicular to the membrane plane. The tilt angle of the alkyl chains in the pure DPPC phase varies significantly in the bilayer, depending on the cholesterol concentration; in the monolayer structures the tilt is distinctly higher, varying little as a function of cholesterol concentration (Table 1).

The position of the Bragg peak of the mixed DPPC:Chol phase changes linearly with the composition ratio. ${ }^{21} \mathrm{We}$ can then deduce from the position of the mixed phase peak in the bilayer at $40 \%$ cholesterol total composition that the DPPC: Chol ratio in the mixed domains is 51:49 mol \%. The position of the Bragg peak along $q_{x y}$ of the mixed DPPC:Chol domain barely shifts when increasing the cholesterol concentration from 40 to $60 \mathrm{~mol} \%$, yielding a composition of $48: 52 \mathrm{~mol} \%$ for the mixed phase. Therefore this is likely to be the nucleation ratio for formation of cholesterol bilayer crystals.

An estimate of the threshold DPPC:Chol ratio required for nucleation of cholesterol may also be made utilizing the Bragg peak intensities. In the previous study that we conducted on SM:Chol mixtures in bilayers, X-ray diffraction from the cholesterol bilayer was observed at both 40:60 and 60:40 SM: Chol ratios. ${ }^{34}$ The composition where cholesterol nucleation occurs was determined then by extrapolating to zero the intensity of the cholesterol Bragg peaks. In the DPPC:Chol system, cholesterol bilayer diffraction was observed only for the 40:60 ratio; therefore no intensity extrapolation can be made. Nevertheless, by assuming a similar dependence of the cholesterol diffraction intensity on composition as in the SM:Chol system, the nucleation ratio is evaluated to be $44: 56 \mathrm{~mol} \%$.

Both methods mentioned above, although based on assumptions, result in similar values of 52 and $56 \mathrm{~mol} \%$. We thus estimate that cholesterol nucleation in DPPC:cholesterol bilayer mixtures occurs at $54 \pm 2 \mathrm{~mol} \%$ cholesterol at $7{ }^{\circ} \mathrm{C}$.

\section{Discussion}

We have compared the structures of crystalline domains and the phases formed in monolayers and bilayers composed of DPPC:cholesterol:POPC mixtures at several cholesterol concentrations. As POPC does not mix well with DPPC and 
Table 1. Crystallographic Data on the Lipid Monolayer and Bilayer Samples ${ }^{a}$

\begin{tabular}{|c|c|c|c|c|c|c|c|c|c|}
\hline & $\begin{array}{l}\mathscr{\mathscr { E }} \\
\frac{\tilde{\Xi}}{2}\end{array}$ & 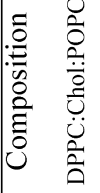 & $\begin{array}{c}d \text {-spacing }[\AA] \\
\pm 0.015\end{array}$ & $\mathrm{UC}[\AA]$ & $\begin{array}{c}\mathrm{A} / \mathrm{mol} \\
{\left[\AA^{2}\right]}\end{array}$ & $\begin{array}{l}\mathrm{PA} \\
{\left[\AA^{2}\right]}\end{array}$ & $\mathrm{CL}[\AA]$ & $\begin{array}{c}\text { Tilt } \\
\text { (along } a \text { ) }\end{array}$ & $\begin{array}{c}\text { Thickness } \\
{[\AA]}\end{array}$ \\
\hline \multirow{6}{*}{$\frac{\overrightarrow{0}}{\bar{m}}$} & \multirow{3}{*}{ 兄 } & 100:0:0 & $\begin{array}{l}d_{\{11\}}=4.37 \\
d_{(02)}=4.24\end{array}$ & $\begin{array}{l}a=5.1 \pm 0.03 \\
b=8.48 \pm 0.05\end{array}$ & $43.3 \pm 0.5$ & 40.4 & $\begin{array}{l}40 \pm 5 \\
100 \pm 15\end{array}$ & $20.9 \pm 1.2$ & $11.3 \pm 0.5$ \\
\hline & & $72: 18: 10$ & $\begin{array}{l}d_{\{11\}}=4.28 \\
d_{(02)}=4.15\end{array}$ & $\begin{array}{l}a=4.99 \pm 0.03 \\
b=8.31 \pm 0.05\end{array}$ & $41.4 \pm 0.3$ & 41.3 & $\begin{array}{l}40 \pm 5 \\
110 \pm 10\end{array}$ & $4.7 \pm 1.2$ & $20.9 \pm 3.1$ \\
\hline & & $54: 36: 10$ & $\begin{array}{l}d_{\{11\}}=4.45 \\
d_{(02)}=4.16\end{array}$ & $\begin{array}{l}a=5.27 \pm 0.03 \\
b=8.32 \pm 0.05\end{array}$ & $43.9+-0.3$ & 43.36 & $\begin{array}{l}240 \pm 20 \\
220 \pm 30\end{array}$ & $8.7 \pm 1.3$ & $19.3 \pm 1.4$ \\
\hline & \multirow{2}{*}{$\stackrel{\substack{\mathscr{d} \\
\Sigma}}{\Sigma}$} & $54: 36: 10$ & $d=4.74$ & & & & $20 \pm 1$ & $0 \pm 4.2$ & $12.3 \pm 0.8$ \\
\hline & & $36: 54: 10$ & $d=4.8$ & & & & $13 \pm 1$ & $0 \pm 3.7$ & $13.4 \pm 0.9$ \\
\hline & Cholesterol & $36: 54: 10$ & $\begin{array}{l}d_{(11)}=6.07 \\
d_{(20)}=5.08\end{array}$ & $\begin{array}{l}a=10 \\
b=7.5\end{array}$ & 37.7 & 36 & $\begin{array}{l}600 \pm 50 \\
590 \pm 50\end{array}$ & 19 & $35 \pm 1.3$ \\
\hline \multirow{4}{*}{$\begin{array}{l}\frac{\overline{0}}{3} \\
\frac{\pi}{0} \\
\stackrel{0}{0}\end{array}$} & \multirow{2}{*}{ 产 } & 100:0:0 & $\begin{array}{l}d_{\{11\}}=4.58 \\
d_{(02)}=4.29\end{array}$ & $\begin{array}{l}a=5.41 \pm 0.03 \\
b=8.59 \pm 0.05\end{array}$ & $46.4 \pm 0.4$ & 41.26 & $\begin{array}{l}90 \pm 30 \\
330 \pm 70\end{array}$ & $27.3 \pm 1.3$ & $17.4 \pm 1.6$ \\
\hline & & $72: 18: 10$ & $\begin{array}{l}d_{\{11\}}=4.55 \\
d_{(02)}=4.28\end{array}$ & $\begin{array}{l}a=5.38 \pm 0.03 \\
b=8.56 \pm 0.05\end{array}$ & $46.0 \pm 0.3$ & 41.46 & $\begin{array}{l}50 \pm 8 \\
50 \pm 5\end{array}$ & $25.7 \pm 1.2$ & $13 \pm 3.5$ \\
\hline & \multirow{2}{*}{ 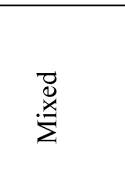 } & $54: 36: 10$ & $d=4.53$ & & & & $19 \pm 1$ & $0 \pm 5.7$ & $12.2 \pm 2.4$ \\
\hline & & $36: 54: 10$ & $d=5.03$ & & & & $22 \pm 2$ & $0 \pm 5.2$ & $10.4 \pm 0.9$ \\
\hline
\end{tabular}

${ }^{a}$ Unit cell dimensions (UC), Area per molecule (A/mol), Projected area (PA), Coherence length (CL). The $\{11\}$ represents both (11) and (1 $\left.\overline{1}\right)$.

cholesterol at $7{ }^{\circ} \mathrm{C}$, we shall refer below, for simplicity, to DPPC:cholesterol proportions within the mixed domains only.

The differences in phase behaviors between the monolayer and bilayer are schematically illustrated in Figure 5. At $20 \mathrm{~mol}$ $\%$ cholesterol, the molecular tilt was found to be lower in the bilayer system relative to the monolayer, but the same DPPC phase is formed in both systems. At $40 \%$ mole cholesterol, the monolayer is composed of one mixed DPPC:Chol phase, whereas in the bilayer phase separation occurs into a mixed DPPC:Chol phase and a DPPC phase. At $60 \mathrm{~mol} \%$ cholesterol, again phase separation occurs in the bilayer, but this time between a mixed DPPC:Chol phase and a cholesterol bilayer crystal. This indicates that the DPPC:Chol miscibility range in symmetrical bilayers is less than $20 \%$.

Interestingly, the DPPC crystalline phase in the bilayer containing 60\% DPPC has sharper peaks relative to the DPPC phases in the bilayer samples with lower cholesterol concentrations; in other words a dramatic increase in the coherence length from $40 \AA$ to $240 \AA$ in the direction of $\boldsymbol{d}^{*}{ }_{\{11\}}$ and from $110 \AA$ to $220 \AA$ in the $\boldsymbol{d}^{*}{ }_{\{02\}}$ direction (see Table 1) is observed, as deduced from the fwhm values (Figure 3c). The 60\% DPPC bilayer has two coexisting phases, which introduces a line tension between their domains. This line tension is likely to drive the system to form larger crystalline domains, by minimizing their boundaries, therefore increasing the coherence lengths. (a) DPPC:Chol:POPC (36:54:10)

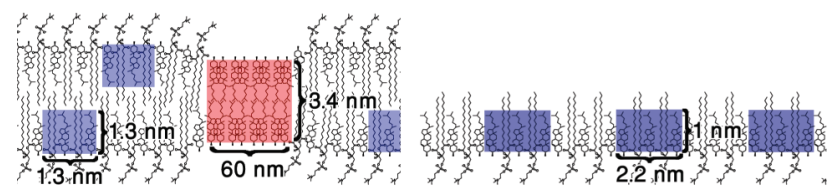

(b) DPPC:Chol:POPC (54:36:10)

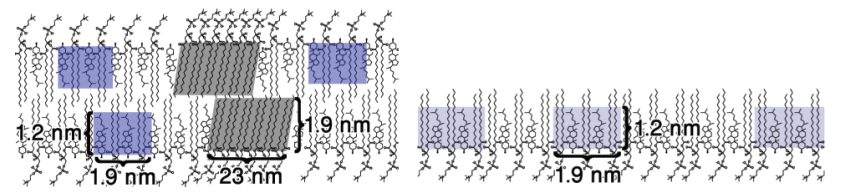

Figure 5. Scheme of (a) bilayer (left) and monolayer (right) of DPPC: Chol (40:60) and (b) bilayer (left) and monolayer (right) of DPPC:Chol $(60: 40)$. POPC was omitted from the scheme. The phase behavior at both compositions in the bilayer differs from the corresponding monolayers. One crystalline mixed phase exists in each monolayer, colored in light blue for DPPC:Chol (60:40) and dark blue for DPPC:Chol (40:60). There is a phase separation in their bilayers. Both bilayers have a similar mixed phase composition which is estimated to be DPPC:Chol ( 50:50). The additional phases are in (a) crystalline cholesterol bilayer (red) and (c) crystalline DPPC phase (gray).

To understand the reason for the observed phase separation of cholesterol in the bilayer system, let us consider first the crystalline behavior of sphingolipids; When spread at the 
Chart 1. Chemical Formulas
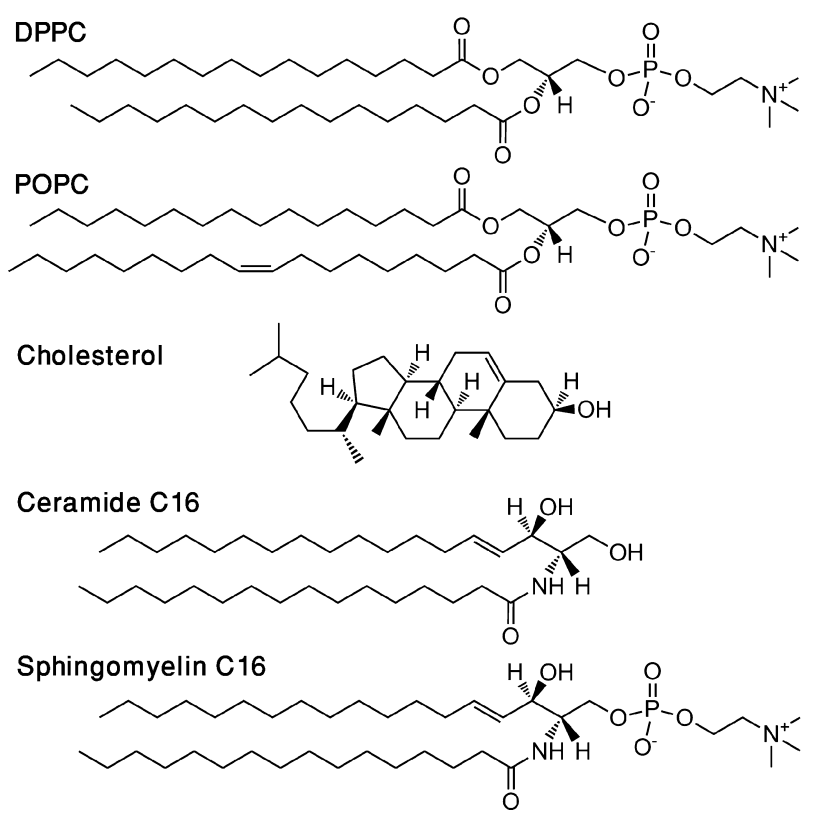

air-water interface, ceramide C16 (Chart 1) spontaneously forms crystalline domains even at negligible surface pressure. ${ }^{24,51}$ Sphingomyelin, however, which consists of a ceramide backbone esterified with a phosphocholine headgroup, does not form crystalline monolayer domains, unless it is subjected to high compression. ${ }^{16,29}$ This lack of self-assembly was attributed to the large hydrophilic headgroup of SM, which disturbs the molecular packing, leading to the "umbrella effect" hypothesis. ${ }^{52}$ A corollary of this concept is that molecules with small head groups such as cholesterol or ceramide readily form mixed phases with SM, because they dilute the interactions between the SM bulky head groups. DPPC is similar to SM in the head group and the alkyl chain length but differs in the backbone chemistry. Unlike SM, DPPC does, however, spontaneously form crystalline domains at the air-water interface at negligible surface pressure.

The driving force of DPPC to form stable crystalline domains suggests that DPPC should tend to phase separate from cholesterol to crystallize at a lower concentration than that of SM, which cannot self-assemble into crystals. This suggestion may explain why, in the bilayers with an overall composition of DPPC:Chol = 60:40, DPPC separates from the DPPC:Chol mixture and forms a pure phase, whereas, in the corresponding bilayers with composition SM:Chol $=60: 40$, no pure SM phase separation is observed. The logic of this argument would suggest similar phase separation behaviors in monolayers composed of mixed DPPC:Chol and SM:Chol. However, as monolayers, both systems have only one crystalline phase at all ratios. The phase separation observed in the bilayers thus must be rather due to interactions between the opposing leaflets.

The cross section of the DPPC molecule is composed of two alkyl chains with an area of $\sim 20 \AA^{2}$ each. The widest average cross section of the rigid moiety of the steroid in the cholesterol molecule is $\sim 38 \AA^{2}$, whereas its branched exocyclic chain has a cross-sectional area of $\sim 28 \AA^{2}$. The crystalline domains formed in the mixed phase in both monolayers and bilayers are

(51) Scheffer, L.; Solomonov, I.; Weygand, M. J.; Kjaer, K.; Leiserowitz, L.; Addadi, L. Biophys. J. 2005, 88 (5), 3381-3391.

(52) Huang, J. Y.; Feigenson, G. W. Biophys. J. 1999, 76 (4), 2142-2157.
10-13 A thick (see Table 1), which corresponds to the length of the steroid moiety only, indicating that the alkyl chains of DPPC are likely to be packed well with the steroid moiety but not with the exocyclic chains.

The crystalline domains in the DPPC monolayer have a chain tilt of $27^{\circ}$, which remains the same when the cholesterol levels are increased to $20 \%$. In the bilayer the tilt is $21^{\circ}$ at $100 \%$ DPPC and is decreased further to $5^{\circ}$ when increasing the cholesterol levels to $20 \%$. We deduce from these data that the interaction of an opposing leaflet in the bilayer is accompanied by interactions between the molecule extremities, presumably by filling the voids between molecules. The thicknesses of all the mixed phase crystalline domains, both monolayer and bilayer, are in the range of $10.4-13.4 \AA$. This thickness indicates that the domains in the opposing leaflets diffract independently and no repetitive structural correlations exist there. Interactions between the leaflets made by partial interdigitation in the crystalline domains are not trivially formed since this would require appropriate alkyl chain orientation and packing registry between the opposing leaflets, demanding, at least, 2-fold symmetry operation. Whether or not the crystalline domains in opposing leaflets are independently arranged or coupled by any short-range correlation between leaflets remains unclear.

In contrast to the mixed phase, partial interdigitation is observed in the cholesterol bilayer crystals. These are formed by virtue of interplay between the lateral packing requirements of the rigid steroid and flexible exocyclic moieties, and the interlayer contact between exocyclic moieties at the interface of the two leaflets, yielding $35 \AA$ thick crystals.

The threshold value at which cholesterol starts forming a crystalline bilayer in DPPC:Chol bilayers is $54 \mathrm{~mol} \%$, which is significantly higher than that in SM:Chol bilayers where cholesterol crystal nucleation occurs at $38 \mathrm{~mol} \%$, measured under the same conditions. The sphingoid backbone of SM has two hydrogen donor groups, hydroxyl and amide, which are not present in the glycerol backbone of DPPC. It is conceivable that the cholesterol hydroxyl group may interact with these groups, thus affecting the positioning of the cholesterol perpendicular to the bilayer surface.

Using molecular dynamics simulations, T. Rog et al. showed that the average distribution of the cholesterol location in one leaflet of the DPPC:Chol bilayer system is $1.6 \AA$ further away from its opposing cholesterol molecule in the other leaflet than in the SM:Chol system. ${ }^{53}$ They also suggested that the DPPC: Chol bilayer is less dynamic in terms of the cholesterol oscillations along the membrane perpendicular axis. The consequence of these differences between the SM:Chol and the DPPC:Chol bilayers is that cholesterol molecules in the former bilayer will more frequently make contact and interdigitate, nucleate, and subsequently form cholesterol bilayer crystals.

The position of the Bragg peak of the mixed DPPC:Chol phase changes linearly with the composition ratio. ${ }^{29}$ We can then deduce from the position of the mixed phase peak in the bilayer at $60 \%$ cholesterol total composition that the DPPC: Chol ratio in the mixed domains is $46: 54 \mathrm{~mol} \%$. Assuming that the system is isotropic and homogeneous, and that the coherence length equals the crystalline domain size, we can calculate by knowing the total lipid composition that the average distance between cholesterol bilayer crystals is $500 \pm 250 \mathrm{~nm}$.

The bilayer cholesterol crystals are not expected to dissolve at body temperatures, although their nucleation threshold in

(53) Rog, T.; Pasenkiewicz-Gierula, M. Biophys. J. 2006, 91 (10), 37563767. 
terms of cholesterol composition is likely to be higher than that at $7{ }^{\circ} \mathrm{C}$. When these bilayers become stacked via interleaving $\mathrm{H}$-bonded water molecules the resulting monoclinic cholesterol monohydrate structure spontaneously transforms into the thermodynamically stable triclinic monohydrate phase, ${ }^{45}$ which incorporates the $12.4 \times 12.4 \AA^{2}$ bilayer motif, and exists as a three-dimensional crystal. Thus, it is conceivable that once formed within the biomembrane the two-dimensional cholesterol crystals, incorporating the $10 \times 7.5 \AA^{2}$ bilayer motif, serve as nucleating domains, which eventually lead to the formation of three-dimensional cholesterol crystals. Cholesterol is found in large amounts at the cytoplasm membrane and can reach levels as high as $45 \mathrm{~mol} \%$ of the total lipid composition. The cholesterol is unlikely to be spread homogeneously, especially because the membrane is largely composed of unsaturated lipids that tend to phase separate from it. Therefore, locally, cholesterol concentrations can be presumably much higher.

\section{Concluding Remarks}

We have monitored here the consequences of interactions between opposing leaflets in a lipid bilayer composed of cholesterol and DPPC separated by immiscible POPC disordered areas. Depending on the lipid composition, the interactions between leaflets led to phase separation, changes in molecular tilt angle, or formation of cholesterol bilayer thick crystals. The cholesterol bilayer crystals begin to form with a DPPC:Chol ratio of $46: 54 \pm 2 \mathrm{~mol} \%$, and the interplay between intra- and interlayer interactions, stabilized by partial interdigitation of the exocyclic chains, leads to bilayer crystallinity. Structural interdigitation was not observed for other phases. Similar phase separation of cholesterol into bilayer crystals was observed also in cholesterol:sphingomyelin bilayer domains at cholesterol concentrations above $38 \%$. We argue that in both systems the nucleation of the cholesterol crystals occurs at concentrations relevant to the actual cell plasma membrane composition. The formation of bilayer thick crystalline domains in the membrane may lead to nucleation of 3D cholesterol crystals under appropriate conditions.

Acknowledgment. We thank HASYLAB for synchrotron beamtime and ELISA: EU financial support of access to synchrotrons/ FELs in Europe. This work was supported by the Israel Science Foundation, and by the Clore Center for Biological Physics. We are grateful to the support of the Helen and Martin Kimmel Center for Nanoscale Science. We thank Dr. Kristian Kjaer for his tremendous help in the development and implementation of the GIXD humidity chamber in beamline BW1.

Supporting Information Available: Complete ref 43. AFM scan of supported lipid monolayer (Figure S1) and un-normalized Bragg peaks of both mono- and bilayer samples (Figure $\mathrm{S} 2$ ). This material is available free of charge via the Internet at http://pubs.acs.org.

JA103975G 